\title{
INVESTIGATION OF THE INFLUENCE OF LIQUID PHASE MOTION ON THE SOLIDIFICATION PROCESS IN THE THREE-DIMENSIONAL REGION
}

\author{
Ewa Wegrzyn-Skrzypczak \\ Department of Mathematics, Czestochowa University of Technology \\ Czestochowa, Poland \\ ewa.skrzypczak@im.pcz.pl
}

Received: 8 January 2021; Accepted: 25 February 2021

\begin{abstract}
In this paper, the numerical model of solidification process with the motion of the liquid phase is presented. The mathematical description of the considered problem is based on the heat conduction equation with convective term and the Navier-Stokes equations with continuity equation. The numerical model uses the Finite Element Method (FEM). The simulations of the solidification process with or without the fluid motion effect are presented and discussed.
\end{abstract}

MSC 2010: $35 K 05,35 K 51,65 M 60$

Keywords: Finite Element Method, numerical modelling, solidification, fluid flow, convection

\section{Introduction}

Choosing the right technology of the casting process, which improves the high-quality of the final product is a difficult and complex challenge, requiring extensive knowledge and practical experience. In the last few decades, the rapid development of information technology has made numerical modelling an important tool used in the selection of casting technology.

Computer simulations of a solidification phenomenon make possible to get the necessary tips and hints to optimize parameters of the real process, and also improve the economic aspects by avoiding the need to prepare many defective prototypes of the casting. Thus, the use of simulation systems becomes an increasingly common and necessary part of manufacturing.

The main purpose of computer simulation is to improve the quality of the casting. Therefore, it is desirable to include in the numerical model as many phenomena accompanying the solidification process as possible. The paper is focused on the investigation of the influence of natural convection of the liquid phase on the formation of the mushy zone as well as its location and shape. 
The effectiveness of numerical modelling is dependent on the complexity of the described physical phenomena and necessary simplifications in computer algorithms. There are many commercial programs (such as MAGMA, PROCAST, NOVAFLOW $[1,2]$ ) offering wide possibilities of simulating foundry processes, however, they are unfortunately expensive. In such a situation, the best solution is to create an authoring program that, thanks to the possibility of modifying the source code can be used in various cases of solidification. The article presents the results of the simulation of the solidification process, considering the motion of the liquid phase, obtained with the use of the proprietary program based on the Finite Element Method.

\section{Mathematical model}

The mathematical model of the analyzed phenomenon is described by the following set of differential equations: the heat transport equation with the convective term (1), the Navier-Stokes equations with buoyancy and Darcy's viscosity terms (2) and the continuity equation (3):

$$
\begin{gathered}
\rho c_{p} \frac{\partial T}{\partial t}+\rho c_{p}\left(v_{i} T_{T_{i}}\right)=\left(\lambda T_{i_{i}}\right)_{\prime_{i}}+\rho_{S} L \frac{d \varepsilon_{S}}{d t} \\
\frac{\partial v}{\partial t}=\frac{\mu}{\rho} v_{i^{\prime} j j}-v_{j} v_{i^{\prime} j}-\frac{1}{\rho} p_{\prime^{\prime}}-\frac{\mu}{\rho K} v_{i}-g_{i} \beta\left(T-T_{r e f}\right) \\
v_{i^{\prime} i}=0
\end{gathered}
$$

where $T[\mathrm{~K}]$ is temperature, $t[\mathrm{~s}]-$ time, $\rho\left[\mathrm{kg} / \mathrm{m}^{3}\right]-$ density, $c_{p}-$ specific heat $[\mathrm{J} /(\mathrm{kgK})], v_{i}[\mathrm{~m} / \mathrm{s}]$ - velocity components, $\lambda[\mathrm{W} /(\mathrm{mK})]$ - coefficient of thermal conductivity, $\rho_{S}\left[\mathrm{~kg} / \mathrm{m}^{3}\right]$ - density of the solid phase, $L[\mathrm{~J} / \mathrm{kg}]$ - latent heat of solidification, $\varepsilon_{S}[-]-$ volume fraction of solid phase $\in[0,1], \mu[\mathrm{kg} /(\mathrm{ms})]-$ dynamic viscosity of liquid phase, $p[\mathrm{~Pa}]$ - pressure, $K\left[\mathrm{~m}^{2}\right]-$ permeability of the porous medium, $g_{i}\left[\mathrm{~m} / \mathrm{s}^{2}\right]-$ components of the gravitational acceleration vector, $\beta\left[\mathrm{K}^{-1}\right]-$ volumetric thermal expansion coefficient, $T_{\text {ref }}[\mathrm{K}]$ - reference temperature.

The Carman-Kozeny relation [3] was used to determine the permeability $K$ of the mushy zone:

$$
K=\frac{d^{2} \varphi^{3}}{180(1-\varphi)^{2}}
$$

where $d[\mathrm{~m}]$ is the average pore diameter in the mushy zone, $\varphi[-]-$ porosity coefficient (in this case $\varphi=1-\varepsilon_{s}$ ). 
The averaged material density was determined as follows:

$$
\rho=\varphi \rho_{L}+(1-\varphi) \rho_{S}
$$

Equations (1)-(3) must be supplemented by appropriate boundary (6-8) and initial conditions (9)-(10):

$$
\begin{gathered}
x, y, z \in \Gamma_{e x t}:-\lambda \mathbf{n} \cdot \operatorname{grad} T=\alpha\left(T-T_{r e f}\right) \\
x, y, z \in \Gamma_{e x t}:-\lambda \mathbf{n} \cdot \operatorname{grad} T=q_{b}=0 \\
x, y, z \in \Gamma_{e x t}: v_{i}=0 \\
t=0:\left.T\right|_{\Omega}=T_{0} \\
t=0:\left.v_{i}\right|_{\Omega}=0
\end{gathered}
$$

where $\alpha\left[\mathrm{W} /\left(\mathrm{m}^{2} \mathrm{~K}\right)\right]$ is the convective heat transfer coefficient, $q_{b}\left[\mathrm{~W} / \mathrm{m}^{2}\right]-$ heat flux on the external boundary, $T_{0}[\mathrm{~K}]-$ initial temperature of the alloy, $\mathbf{n}[-]-$ vector normal to the external boundary.

\section{Numerical model}

The FEM $[4,5]$ equations for the heat transport equation with the convective term (1) were derived using Weighted Residual Method (WRM) [6, 7]. In order to provide the stable numerical solution of equation (1), the Streamline Upwind Petrov-Galerkin (SUPG) method $[8,9]$ was used at the stage of spatial discretization of the considered domain. The global set of the FEM equation was obtained as a result of the backward Euler time-integration scheme and the aggregation procedure:

$$
\left(\mathbf{K}+\mathbf{A}+\frac{1}{\Delta t} \mathbf{M}\right) \mathbf{T}^{f+1}=\frac{1}{\Delta t} \mathbf{M} \mathbf{T}^{f}+\mathbf{B}
$$

where $\mathbf{K}$ denotes the heat conductivity matrix, $\mathbf{A}$ - convection matrix, $\mathbf{M}-$ heat capacity matrix, $\mathbf{T}^{f}, \mathbf{T}^{f+1}$ - vectors of unknowns temperatures for time $f, f+1$, B - right hand side vector containing boundary conditions, $\Delta t$ - time step.

The numerical solution of the Navier-Stokes equations is more difficult to obtain due to the pressure gradient, which in the case of incompressibility makes the global coefficient matrix singular. For this reason equation (2) was solved with the use of Characteristic Based Split (CBS) method. This method is based on the Chorin's projection method developed in 1968 [10] and then described and expanded by Taylor, Zienkiewicz and Codina $[7,11]$. 
According to the CBS method, equations (2) was initially solved without a pressure term. As a result of this procedure, incorrect velocity components were obtained. To solve this problem, an artificial velocity field $\mathbf{V}^{*}$ was introduced. Correction of the velocity field was performed after calculation of the pressure field. Pressure was determined from Poisson's equation, which finally has the following form:

$$
\mathbf{p}^{f+1}=\frac{\rho}{\Delta t} \mathbf{G}^{T} \mathbf{V}^{*} \cdot \mathbf{K}_{p}^{-1}
$$

where $\mathbf{G}$ is the gradients matrix.

Using the known pressure field $\mathbf{p}^{f+1}$, the velocity correction $\Delta \mathbf{V}^{*}$ is calculated:

$$
\Delta \mathbf{V}^{*}=-\frac{\Delta t}{\rho} \mathbf{M}^{-1} \mathbf{G p}^{f+1}
$$

finally the real velocity field in the step $f+1$ is obtained:

$$
\mathbf{V}^{f+1}=\mathbf{V}^{*}+\Delta \mathbf{V}^{*}
$$

The algorithm of the consecutive calculations performed in each time step can be written as follows:

1. For the known values of $\mathbf{T}^{f}$ and $\mathbf{V}^{f}$, the temperature field $\mathbf{T}^{f+1}$ is calculated.

2. For determined $\mathbf{T}^{f+1}$, the velocity field $\mathbf{V}^{*}$ is calculated.

3. For determined $\mathbf{V}^{*}$, the pressure field $\mathbf{p}^{f+1}$ is calculated.

4. For determined $\mathbf{p}^{f+1}$, the correction of the velocity field $\Delta \mathbf{V}^{*}$ is calculated.

5. The velocity field $\mathbf{V}^{f+1}$ is obtained as a sum $\mathbf{V}^{*}$ and $\Delta \mathbf{V}^{*}$.

6. The velocity field $\mathbf{V}^{f+1}$ and the temperature filed $\mathbf{T}^{f+1}$ are treated as $\mathbf{V}^{f}$ and $\mathbf{T}^{f}$, return to point 1 .

\section{Example of calculations}

On the basis of theoretical assumptions, the authoring computer program using FEM was made. A simulation of solidification process of the Fe-C alloy was carried out, considering the movement of the liquid and ignoring the movement of the solid. The main goal of the computer simulation was to show the process of solid phase growth in the casting and the convective movements accompanying this phenomenon in the liquid and solid-liquid phases.

The geometry of considered area was presented in Figure 1. The cuboid was filled with the Fe-C alloy, the material properties of which are presented in Table 1. A GMSH generator was used to create finite element mesh consisting of 22367 nodes and 123124 tetrahedrons. 


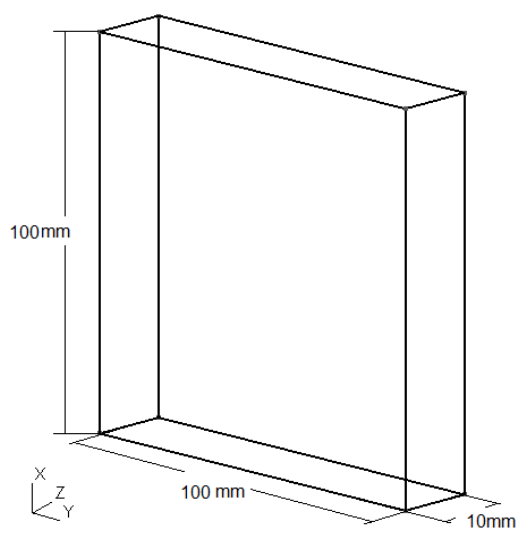

Fig. 1. Geometry of the considered area

Table 1. Material properties of Fe-C alloy used in calculations

\begin{tabular}{|l|c|c|}
\hline \multicolumn{1}{|c|}{ Material parameter } & Liquid phase & Solid phase \\
\hline Thermal conductivity $\lambda[\mathrm{W} / \mathrm{m} \mathrm{K}]$ & 23 & 35 \\
\hline Density $\rho\left[\mathrm{kg} / \mathrm{m}^{3}\right]$ & 6915 & 7800 \\
\hline Specific heat $c_{p}[\mathrm{~J} / \mathrm{kg} \mathrm{K}]$ & 837 & 644 \\
\hline Dynamic viscosity $\mu[\mathrm{kg} / \mathrm{m} \mathrm{s}]$ & $6.94 \cdot 10^{-3}$ & $\rightarrow \infty$ \\
\hline Volumetric thermal expansion coefficient $\beta\left[\mathrm{K}^{-1}\right]$ & $2.7 \cdot 10^{-4}$ & - \\
\hline Liquidus temperature $T_{L}[\mathrm{~K}]$ & \multicolumn{2}{|c|}{1766} \\
\hline Solidus temperature $T_{S}[\mathrm{~K}]$ & \multicolumn{2}{|c|}{$2.7 \cdot 10^{5}$} \\
\hline Latent hcat of solidification $L[\mathrm{~J} / \mathrm{kg}]$ & \multicolumn{2}{|c|}{} \\
\hline
\end{tabular}

The boundary and initial conditions for both cases of calculations are presented in Figure 2. The time step in both calculation variants was constant $\Delta t=0.005 \mathrm{~s}$.

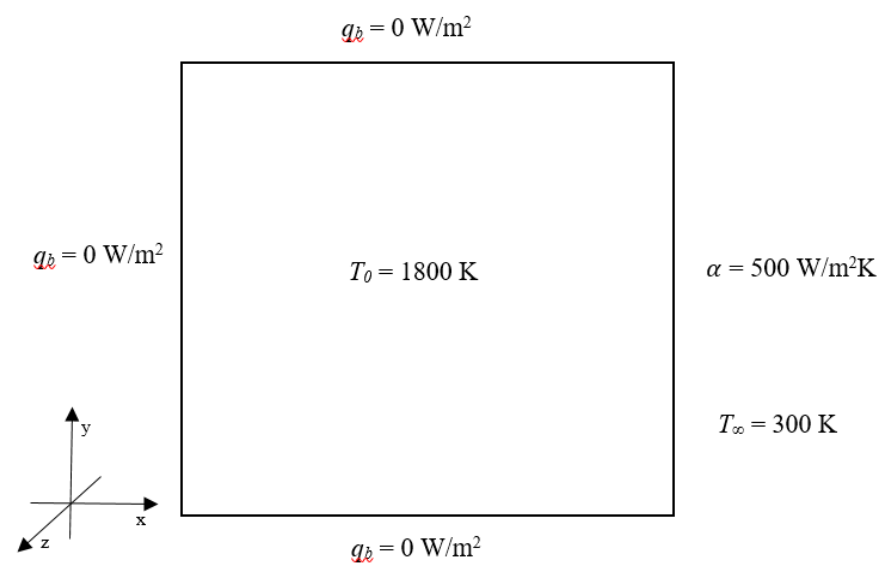

Fig. 2. The boundary and initial conditions used in calculations 
In Figure 3a-d, the amount of the solid phase as well as the temperature field for solidification process with and without convection for $10 \mathrm{~s}$ are presented. At this initial stage, significant differences in the distribution of these quantities can be noticed. These differences are observed as the result of the liquid movement caused by the change of the temperature due to heat transport between the right external boundary and the environment. The maximum velocity at this moment equals $0.047 \mathrm{~m} / \mathrm{s}$ (Fig. 5a).

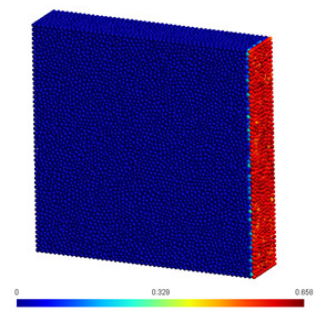

(a)

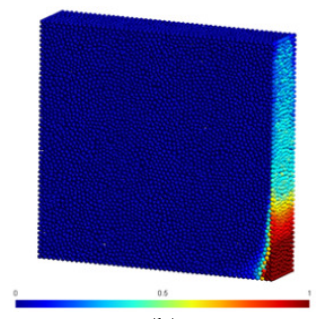

(b)

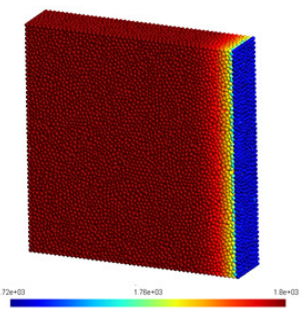

(c)

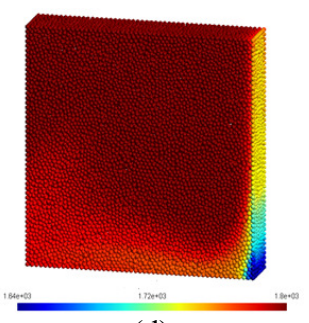

(d)

Fig. 3. a) Solid phase fraction during solidification process without convection for $10 \mathrm{~s}$

b) Solid phase fraction during solidification process with convection for $10 \mathrm{~s}$

c) Temperature field during solidification process without convection for $10 \mathrm{~s}$

d) Temperature field during solidification process with convection for $10 \mathrm{~s}$

After 20 seconds of the start of solidification process, the solid phase builds up in the lower right corner of the cuboid (Fig. 4b). Its occurrence affects the velocity field in the liquid part of the casting (Fig. 5b).

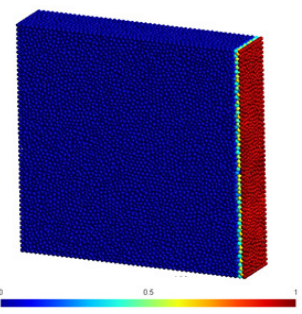

(a)

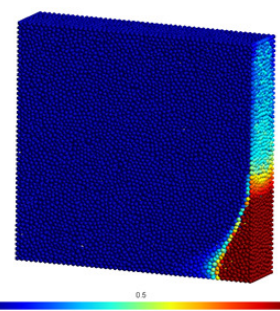

(b)

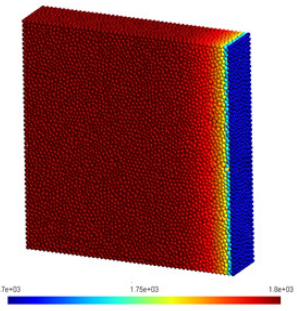

(c)

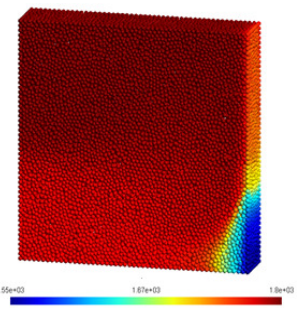

(d)

Fig. 4. a) Solid phase fraction during solidification process without convection for $20 \mathrm{~s}$

b) Solid phase fraction during solidification process with convection for $20 \mathrm{~s}$

c) Temperature field during solidification process without convection for $20 \mathrm{~s}$

d) Temperature field during solidification process with convection for $20 \mathrm{~s}$

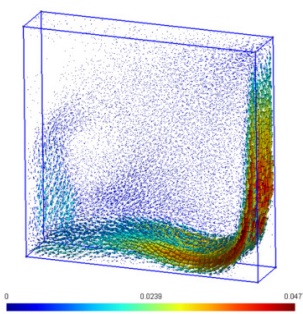

(a)

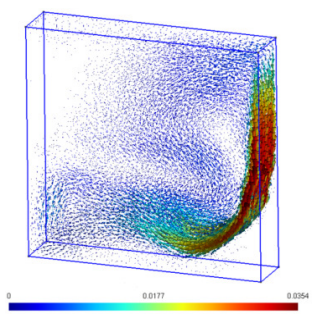

(b)

Fig. 5. Velocity field for: a) $10 \mathrm{~s}$, b) $20 \mathrm{~s}$ 
In Figure 6a-d, the amount of the solid phase and the temperature field for $80 \mathrm{~s}$ are presented. In Figure 6b, one can observe the mushy zone formation in the lower part of the cuboid. The mushy zone is also clearly visible during analysis of the velocity field (Fig. 9a).

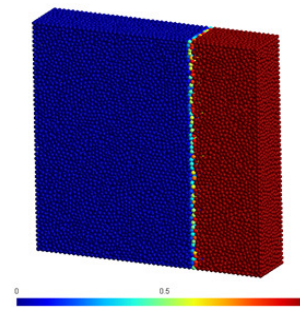

(a)

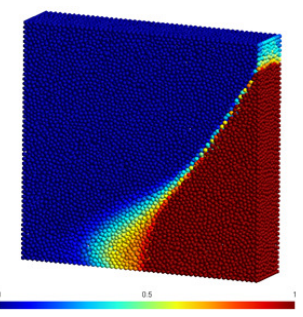

(b)

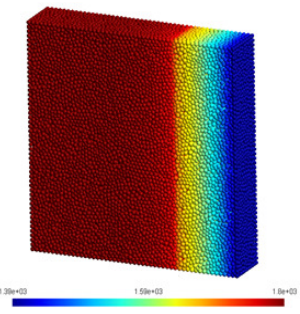

(c)

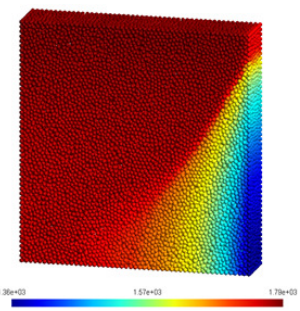

(d)

Fig. 6. a) Solid phase fraction during solidification process without convection for $80 \mathrm{~s}$

b) Solid phase fraction during solidification process with convection for $80 \mathrm{~s}$

c) Temperature field during solidification process without convection for $80 \mathrm{~s}$

d) Temperature field during solidification process with convection for $80 \mathrm{~s}$

The advanced stage of solidification is presented in Figure $7 \mathrm{a}-\mathrm{d}$. The velocities slowly vanish which is shown in Figure $9 b$.

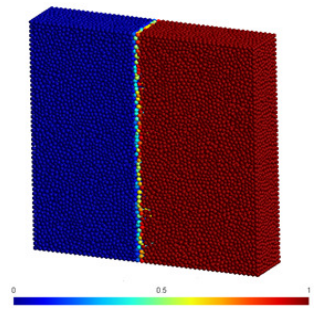

(a)

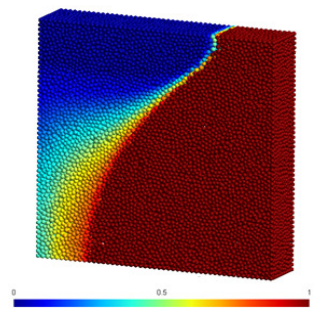

(b)

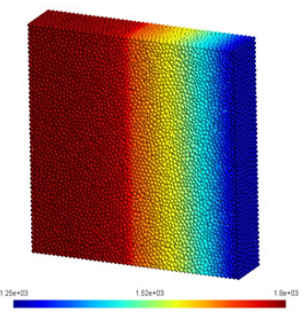

(c)

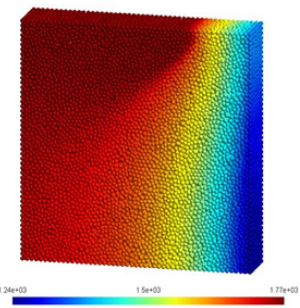

(d)

Fig. 7. a) Solid phase fraction during solidification process without convection for $160 \mathrm{~s}$

b) Solid phase fraction during solidification process with convection for $160 \mathrm{~s}$

c) Temperature field during solidification process without convection for $160 \mathrm{~s}$

d) Temperature field during solidification process with convection for $160 \mathrm{~s}$

Figure 8a-d shows the end stage of solidification process. One can observed, that solidification process lasts longer in the absence of convective movement of the liquid (Fig. 8a-b). In the case of the occurrence of convective movements of the liquid for $320 \mathrm{~s}$, the velocities are almost zero (the liquid moves at a speed of $0.00048 \mathrm{~m} / \mathrm{s}$ ) as shown in Figure 9c.

Analyzing the velocity fields for the selected moments, one can notice that the influence of convective movements on the formation of the solid phase in the casting is quite significant. Without it, the solid phase grows uniformly over the cuboid starting with the cooled wall. If natural convection is taken into account in the model, it affects the process of solid phase growth. It can be observed that the solid phase accumulates in the lower right corner of the casting. 


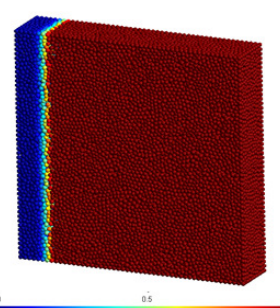

(a)

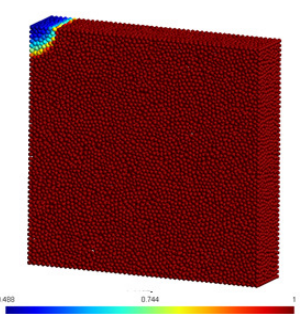

(b)

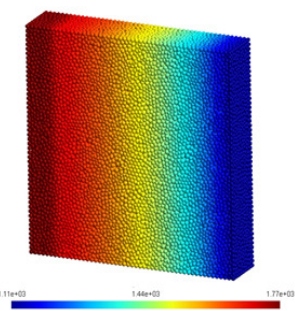

(c)

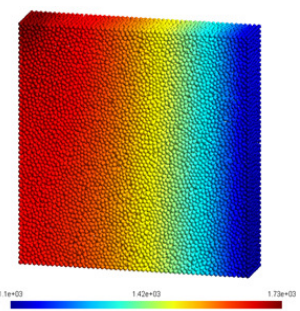

(d)

Fig. 8. a) Solid phase fraction during solidification process without convection for $320 \mathrm{~s}$

b) Solid phase fraction during solidification process with convection for $320 \mathrm{~s}$

c) Temperature field during solidification process without convection for $320 \mathrm{~s}$

d) Temperature field during solidification process with convection for $320 \mathrm{~s}$

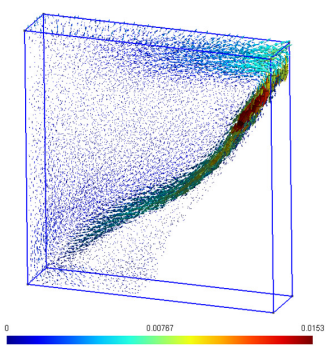

(a)

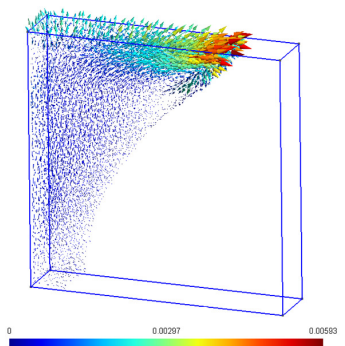

(b)

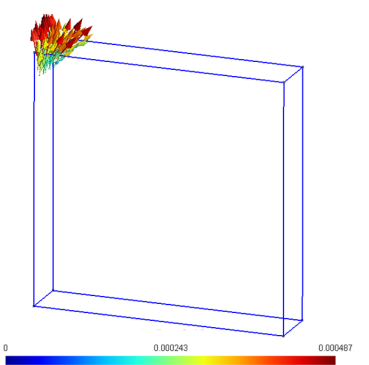

(c)

Fig. 9. Velocity field for: a) $80 \mathrm{~s}$, b) $160 \mathrm{~s}$, c) $320 \mathrm{~s}$

\section{Conclusions}

Numerical modelling of solidification process with and without the movement of the liquid phase makes it possible to show the impact of the convective motion on the evolution and shape of the mushy zone and the distribution of the solid fraction in the casting. The growth of the solid phase in the mushy zone significantly affects the damping of the liquid movement. In turn, the movement of the liquid slightly reduces the time of solidification. Taking into account the movement of the liquid in solidification process makes it possible to more accurately predict the distribution of impurities, e.g. carbon, sulphur, etc., and this is the main purpose of the future work. The modifications of the created original solver are easier to do, then in the case of expensive commercial packages.

\section{References}

[1] Vasková, I., Fecko, D., \& Eperješi, L. (2011). Comparison of simulation programs MAGMASOFT and NOVAFLOW\&SOLID in terms of results accuracy. Archives of Foundry Engineering, 11, 1, 51-54.

[2] Khan, M.A.A., \& Sheikh, A.K. (2018). A comparative study of simulation software for modelling metal casting processes. International Journal of Simulation Modelling, 17(2), 197-209. 
[3] Kaviany, M. (1991). Principles of Heat Transfer in Porous Media. New York: Springer-Verlag.

[4] Zienkiewicz, O.C. (1977). The Finite Element Method. London: Mc Graw-Hill.

[5] Majchrzak, E., Mochnacki, B. (2004). Numerical Methods. Theory, Numerical Aspects, Algorithms. Gliwice: Publication of the Silesian University of Technology.

[6] Bathe, K.J. (1982). Finite Element Procedures in Engineering Analysis. Prentice-Hall.

[7] Zienkiewicz, O.C., Taylor, R.L. (2000). The Finite Element Method. Volume 1-3: Fluid Dynamics. Butterworth \& Hienem.

[8] Bochev, P. (2001). Finite Element Methods Based on Least Squares and Modified Variational Principles. COMP2PMAC Lecture Notes, Postech, Pohang, South Korea.

[9] Hughes, T.J.R. (1987). Recent progress in the development and understanding of SUPG methods with special reference to the compressible Euler and Navier-Stokes equations. International Journal for Numerical Methods in Fluids, 7, 1261-1275.

[10] Chorin, A.J. (1968). Numerical solution of the Navier-Stokes equation. Mathematics of Computation, 23, 745-762.

[11] Zienkiewicz, O.C., Codina, R. (1995). A general algorithm for compressible and incompressible flow, Part I. The split characteristic based scheme. International Journal for Numerical Methods in Fluids, 20, 869-885. 